\title{
EL PENSAMIENTO DOCENTE Y SU INFLUENCIA EN LA IMPLANTACIÓN DE LAS TECNOLOGÍAS DE LA INFORMACIÓN Y LA COMUNICACIÓN EN EL AULA: DESAFÍOS Y OPORTUNIDADES
}

\author{
Javier Gómez-López \\ Jacobo Cano Escoriaza \\ Universidad de Zaragoza
}

\begin{abstract}
RESUMEN: Un reto fundamental de la educación actual es la implantación de las tecnologías de la información y comunicación (TICs de aquí en adelante) en las instituciones educativas para mejorar la gestión y especialmente, la calidad del proceso de enseñanza-aprendizaje. Este artículo pretende analizar las dificultades y obstáculos -primarios y secundarios- existentes en su implantación, focalizando la atención en el pensamiento docente al considerarse un factor clave.

Se parte de un análisis general de la estructura y funcionamiento del pensamiento docente centrado en las actitudes, creencias y expectativas, valorando su influencia en la integración de las TICs. Las conclusiones apuntan la necesidad de dotar a los docentes de una visión positiva ante el uso de las TICs en una serie de propuestas que faciliten su progresiva implantación.
\end{abstract}

ABSTRACT: The fundamental challenge of nowadays education is the implementation of information technologies and communication technologies (ICT shereafter) in the educational institutions in order to improve the managing and specially, the teaching-learning process quality. This article aims to analyze the reasons and barriers - primary and secondary ones- that exists in their implementation, focusing attention on the teacher thought of being considered a key factor.

It starts on a general analysis of the structure and functioning of teacher thought focused on attitudes, beliefs and expectations, it assessing their influence on the integration of ICTs. The findings aim the need to give teachers a positive outlook towards the use of ICTs in a series of proposals that facilitate it implementation gradually.

PALABRAS CLAVE: Pensamiento docente, Tecnologías de la información y de la comunicación, profesorado, actitudes, creencias.

KEYWORDS: Teaching thought, Information and Communications Technologies, teachers, attitudes, beliefs. 


\section{INTRODUCCIÓN: HACIA UNA CONTEXTUALIZACIÓN DE LA IMPLANTACIÓN DE LAS TICS EN EL AULA}

Una cuestión clave es conocer las posibilidades de las TICs en las aulas para mejorar la calidad del proceso de enseñanza-aprendizaje, para lo que nos parece relevante clarificar la conceptualización tal y como señala González (1998), como "el conjunto de herramientas, soportes y canales para el tratamiento y acceso a la información, que generan nuevos modos de expresión, nuevas formas de acceso y nuevos modelos de participación y recreación cultural". Una característica específica de la cambiante sociedad actual, denominada de la información (Marqués, 2008), es la fácil obtención de todo tipo de datos e imágenes de los que disponemos. El conocimiento evoluciona a una velocidad asombrosa, siendo fácil e instantáneo el acceso, gracias al uso y expansión de las TICs.

Se constata que un número creciente de los adolescentes forma parte de una generación que ha nacido en la era digital, con un lenguaje que tienen asumido y que condiciona su cosmovisión, son "nativos digitales". Por otro lado, los adultos se encuentran en una sociedad distinta a la analógica en la que se formaron, siendo por lo tanto "inmigrantes digitales". La constante evolución de las TIC puede incrementar la distancia entre ellos agrandando la denominada brecha digital (Prensky, 2001). En la actualidad, consideramos clave el papel del profesorado, como agentes de cambio y colaboradores en la transformación social, para reducir las diferencias en dicha brecha y reducir el analfabetismo digital (Gilster, 1997), posibilitando una mayor igualdad de oportunidades y cohesión social.

En la sociedad actual, el conocimiento de los elementos básicos de las TICs es necesario, pero no suficiente. En este sentido, el profesorado, a la luz de las reformas educativas en España, está participando en el debate de considerar el papel más o menos relevante que tienen, por ejemplo, los procesos de memorización, que previamente ha sido un pilar indiscutible del proceso educativo, frente a la necesidad de desarrollar las habilidades, capacidades y competencias personales o "capacidad de actuar de manera eficaz en un tipo definido de situación, capacidad que se apoya en conocimientos, pero que no se reduce a ellos" (Perrenoud, 2002:7). El desarrollo de competencias en los educandos va en la línea de potenciar el pensamiento crítico, la competencia tecnológica, la iniciativa o la colaboración (Angeli \& Valanides, 2009) que permitan su emancipación.

Nos encontramos más próximos, por lo tanto, en la sociedad actual a llevar a cabo la necesaria renovación metodológica para esta concepción del aprendizaje, que, por otra parte, requiere una formación integral permanente a lo largo de toda la vida (Lifelong Learning), donde las TICs pueden desarrollar un papel clave. Además de la necesidad de su conocimiento y uso y debido a la flexibilidad que presentan, suponen una de las mejores herramientas de la educación permanente, al ser un recurso que, por un lado, ofrece múltiples posibilidades metodológicas a todos los niveles, como por otro, permite superar las dificultades espacio-temporales o de nivel que existen (Esteban, 2005).

Además de lo reseñado, se recoge el conjunto de razones que justifican su utilización en el proceso de enseñanza-aprendizaje, entre las que destaca "la aparición de nuevos canales de comunicación respecto a los cuales desconocemos sus ver- 
daderas potencialidades para el aprendizaje" (Cabero, 2001:2). Ademas, como se recoge en los planteamientos del Espacio Europeo de Educación Superior una nueva educación es necesaria bajo el amparo de las TICs, ya que:

“Existe una necesidad de innovación y reforma que va mucho más allá de instalar meros programas de mejora o aprendizaje a través de la red, ya que requiere cambios estructurales en la concepción de la educación y en la adaptación a esta nueva situación social provocada del desarrollo tecnológico e informático" (Gómez, Mur \& Sanz, 2010:2).

En este contexto, la educación se encuentra en un periodo de redefinición y renovación a todos los niveles, buscando la transformación y adecuación del proceso de enseñanza-aprendizaje a la realidad mencionada (Sefton-Green, 2006). Desde nuestro punto de vista, este desarrollo pasa por la implementación progresiva de una pedagogía constructivista, que recoge como característica fundamental ser un proceso formativo centrado en el alumno. Él es el protagonista de su proceso de enseñanza-aprendizaje gracias al desarrollo de un pensamiento reflexivo que se aprovecha de la construcción social del conocimiento por medio de la interacción, y eso es algo que potencian las TICs (Hughes \& Zachariah, 2001; Otto \& Albion, 2002). Resulta valioso considerar que el ordenador tiene el potencial necesario para, mediante su utilización como herramienta cognitiva, apoyar todas las mencionadas características en un curriculum centrado en el estudiante, motivador y dinámico. (Hawkride, 1990; LaJoie, 2002).

Esta nueva pedagogía requiere de una serie de cambios en el aula, desde los principios motivadores del curriculum, los agentes que lo establecen, las estrategias metodológicas y de evaluación así como los productos del mercado. Todas estas premisas abocan hacia un nuevo rol docente en el que pase a asumir como funciones la planificación y desarrollo de acciones formativas; el diseño, producción y evaluación de contenidos y por último, el conocimiento de los diversos medios disponibles (Gisbert, 2000:13). Con ello se pretende, como indica dicha autora, "el desarrollo de nuevas estrategias de enseñanza basadas más en la capacidad de comunicación y secuenciación y selección de contenidos que no en la transmisión masiva de información y de contenidos. Información no es lo mismo que conocimiento".

Este aprendizaje realizado mediante las TICs, contiene una serie de características recogidas por varios autores (Li-Ping \& Austin, 2009), entre las que destaca su efectividad, ya que presenta diversas ventajas, como las ofrecidas por los entornos de e-learning o las redes sociales como el Twitter, pero sin olvidar cómo ayudan a los estudiantes que presentan necesidades específicas de apoyo educativo (Cabero, 2008). Esta nueva forma de interacción entre docente y discente, que puede ser sincrónica o asíncrona, permite un proceso de enseñanza-aprendizaje, que, como se ha mencionado, el tiempo y el lugar dejan de ser dificultades insalvables. La comunidad educativa puede establecer, por lo tanto, una conexión común mediante el intercambio de conocimientos, experiencias e ideas, aun cuando los miembros no puedan interactuar físicamente. Sin embargo, estos autores también reseñan que presenta puntos negativos, como el posible deterioro del aprendizaje intrínseco al depender de un elemento extrínseco como es el ordenador o el acceso a Internet. 
Destacamos las potencialidades de las Tics remarcando el papel que juegan en las instituciones educativas al tener éstas como misión esencial la preparación para la vida, y ésta, en la actualidad, es cada vez más tecnológica. Los docentes van siendo más conscientes de estas potencialidades de las Tics para la educación integral de los alumnos mediante el desarrollo de las competencias (Iglesias, Veiga \& González, 2000), pero nos parece relevante señalar diversos factores que están ralentizando la implantación de éstas y que se señalan a continuación.

\section{BARRERAS QUE DIFICULTAN EL PROCESO DE IMPLANTACIÓN DE LAS TIC EN EDUCACIÓN}

Existen una serie de variables interrelacionadas que impiden la implantación de esta innovación educativa. Estas barreras actúan desde diferentes niveles y ámbitos orientadas a determinados agentes del proceso innovador. Su estudio tiene como objetivo centrar la atención en: describir e interpretar los múltiples elementos a considerar para su reducción, análisis y comprensión, ya que, como se refleja, son multitud de elementos a considerar y tener en cuenta. Las barreras centran su atención fundamentalmente en las instituciones educativas y/o políticas en el caso de las de primer orden, mientras que las de segundo orden se fundamentan o centran su interés en el docente, responsable último de la innovación educativa en el aula.

Diversos autores (Veen, 1993; Rosen \& Weil, 1995; Ertmer, 1999; Snoeyink \& Ertmer, 2001; Ching \& Huang, 2008) reflejan una categorización de los mismos, pudiéndose agrupar de la siguiente manera:

Tabla 1. Barreras para la implantación de las TIC

\begin{tabular}{|l|l|l|}
\hline \multicolumn{3}{|c|}{ Barreras para la implementación de las TICs } \\
\hline Tipo & $\begin{array}{l}\text { Primer orden o institucionales, } \\
\text { externas. }\end{array}$ & $\begin{array}{l}\text { Segundo orden o individuales, } \\
\text { internas. }\end{array}$ \\
\hline Concepto & $\begin{array}{l}\text { Barreras surgidas en el seno } \\
\text { organizativo o institucional, agentes } \\
\text { externos al intercambio directo de } \\
\text { enseñanza aprendizaje, comunes a } \\
\text { todos los implicados. }\end{array}$ & $\begin{array}{l}\text { Barreras surgidas en el propio } \\
\text { agente del proceso de enseñanza- } \\
\text { aprendizaje: el docente, son, por lo } \\
\text { tanto, específicas e individuales. }\end{array}$ \\
\hline Característica \\
principal & $\begin{array}{l}\text { Constante evolución de la misma } \\
\text { forma que las TICs, surgen y } \\
\text { desaparecen barreras constantemente. }\end{array}$ & $\begin{array}{l}\text { Barrera principal del proceso, por si } \\
\text { sola puede evitar el proceso o influir } \\
\text { en laparición de las de primer } \\
\text { orden. }\end{array}$ \\
\hline Necesidad & $\begin{array}{l}\text { Tiempo, recursos, confianza, } \\
\text { resiliencia, organización o soporte } \\
\text { técnico necesario para dar solución } \\
\text { a la posible presencia de problemas } \\
\text { técnicos }\end{array}$ & $\begin{array}{l}\text { Tiempo, acceso a los recursos } \\
\text { informáticos de calidad, } \\
\text { entrenamiento efectivo, percepción } \\
\text { positiva de las TICs así como las } \\
\text { tecno-actitudes de los docentes. }\end{array}$ \\
\hline
\end{tabular}

Fuente: elaboración propia. 
Esta complejidad avistada implica que múltiples investigaciones se han realizado sobre el proceso, pero son necesarias más aún para desarrollar las soluciones ante las diversas barreras que se conocen y prevenir las que pueden surgir, mostrando el camino que conduce hacia una eficiente y progresiva escuela digital.

$\mathrm{Al}$ analizar las barreras de primer orden o institucionales, destacamos que la integración de las TIC no es una tarea sencilla debido a múltiples factores que entorpecen su presencia, ya que como refleja la literatura, las nuevas tecnologías se infrautilizan en el aula (Sutherland et al, 2004; Al-Senaidi, Lin \& Poirot, 2009). Los factores recogidos en este tipo de barreras de primer orden o también denominadas ambientales, presentan una importancia fuera de toda duda, (Mueller Wood, Willoughby, Ross \& Specht, 2008). Si bien es cierto que los problemas en principio se solventan mediante la aplicación de diversos recursos, tanto materiales como humanos, también se reconocen problemas de carácter técnico y organizativo de más compleja respuesta (Doomekamp, 1994).

La implantación de las TICs se complejiza con su constante evolución y con los diferentes cambios instucionales en la escuela, lo que implica la desaparición de antiguas barreras y la creación de otras nuevas fruto de las necesidades creadas ante tal innovación. Un ejemplo de ello es la necesidad actual de contar con una red de Internet de alta velocidad en el centro, a ser posible con acceso remoto (Mueller et al, 2008). Esto es así porque comienzan desde el más alto nivel, en el papel que desempeñan las volubles políticas tanto nacionales como locales en la implantación de las TICs (Tondeur, Keer, Braak y Valcke, 2008), dando lugar a dos escalas: barreras generales o nacionales y locales.

En las generales o nacionales, se ve desde la incoherencia existente entre el uso de las TIC y el curriculum establecido en la actualidad en diversos países, como en EE.UU. (Tondeur et al, 2008), a la necesaria implantación en el curriculo de la formación a docentes necesaria para su desempeño. (Gialamas \& Nikolopoulou, 2010). Queda patente, por lo tanto, la necesidad de mantener una política directa y constante, para evitar confusiones e inseguridad a los docentes (Mueller et al, 2008).

En las locales, por su parte, los autores focalizan la atención en la necesidad de considerar el trabajo con las TIC en diversas escuelas (Baylor \& Ritchie, 2002; Tang \& Ang, 2002); la necesidad de un proceso continuo de calidad en todas las prácticas institucionales (MacBeath, 1999), y la necesaria colaboración entre los diferentes centros e instituciones que favorezca su uso (Triggs \& John, 2004).

Por último, se destaca la importancia de la cultura escolar en la integración de las TICs, entendida como las normas y valores asumidos por la institución, ya que afecta e implica la organización de la misma, (Devos, Bouckenooghe, Engels, Hotton, \& Aelterman, 2007). Es muy importante debido a que en un centro en el que se considere una pérdida de tiempo, condiciona mucho cualquier uso o práctica que se derive de ellas (Tondeur et al, 2008).

Los diferentes estudios realizados analizan el proceso desde una óptica que, desde nuestro punto de vista puede mejorarse, ya que existen numerosas referencias que analizan la resistencia de las escuelas al cambio como el problema que evita la integración de las TICs, cuando éste se encuentra en la ausencia de experiencias educativas positivas con su uso. Los centros no buscan resistirse, sino conocer diferentes formulas 
contrastadas que les ayude a proporcionar una acción educativa de calidad en un proceso tan innovador como complejo para los centros. (Hulpia \& Valcke, 2004).

Todas estas barreras no hacen sino complicar un procedimiento que, por otra parte, debería ser sencillo debido a las altas motivaciones que implica, educativamente, tener una herramienta que te permite mejorar el proceso de enseñanzaaprendizaje. Por si fuera poco, su evolución y cambio no facilita las cosas, aunque progresivamente se van solucionando. Aun así y con la desaparición progresiva de todas, todavía permanecen barreras, si cabe, aún más altas para superar, las de segundo orden.

Estas barreras de segundo orden o personales tienen una gran relevancia sobre el pensamiento docente, ya que, por un lado, son un bastión importante y decisivo de cara a la integración de las TICs en el aula, -aunque desaparecieran las de primer orden-, y por otro, por su carácter, ya que requieren de la percepción positiva o valiosa de la tecnología por parte de los docentes pudiendo, por sí mismas, reducir los efectos percibidos de las variables externas (Ching \& Huang, 2008)

Esta potencialidad contrasta, desde nuestro punto de vista, con la poca presencia de análisis crítico a nivel metodológico y evaluativo sobre el aprendizaje con y a través de las TIC que se le proporciona al docente. De esta forma, se le prestan pocas facilidades para descubrir e integrar las ventajas que presentan éstas. Al no tenerlas, su uso y el esfuerzo que requieren, son percibidos como algo inutil por parte del docente, lo que deriva en que no se dedica el tiempo y esfuerzo necesario al aprovechamiento e integracion de las TICs (Caracena, 2007)

Para ello, es necesario que los docentes puedan vivenciar experiencias positivas en el uso de las TICs que les anime a desarrollar el potencial de las mismas y por lo tanto modificar sus pensamientos (Abrami, 2001).Ese cambio no podrá desarrollarse si no se encuentra un nivel de autonomía básico y necesario en cada centro para el desarrollo de prácticas escolares y una colaboración educativa innovadora (Tondeur et al, 2008).

El docente y sus percepciones es el factor más importante (van Braal et al, 2004) para implementar diferentes innovaciones utilizando las TICs debido a que tienen el contacto directo con los estudiantes y experimenta personalmente las barreras y ayuda a las soluciones (Mueller et al, 2008). Los docentes se preocupan por las modificaciones derivadas de la implantación de las TICs a los medios educativos y las repercusiones que esto puede tener sobre su rol y vida profesional, influyendo sobremanera al proceso, debido a que las valoraciones de los docentes pueden incluso trasladarse a la valoración de los alumnos (Iglesias et al, 2000).

Este traspaso es recogido, en función del determinismo recíproco de Bandura (1987), en un modelo de creencias del docente en el que su pensamiento sobre un campo, -como pueden ser las TICs-, puede determinar en gran medida su comportamiento en el aula. Esto puede, de igual modo, retroalimentar las creencias mediante un procedimiento de auto-atribución, de forma que los pensamientos docentes, los comportamientos en el aula y la situación escolar se influyen mutuamente en un proceso recíproco.

Teniendo todo ello en cuenta, queda patente la necesidad de contar principalmente con el análisis del pensamiento del docente ante la inclusión de cualquier innovación educativa en el aula. Es el principal agente de cualquier innovación a 
implantar en el aula y por lo tanto el que, en última instancia, va a contribuir al éxito o al fracaso de la integración de las TICs, debido a que las estas no son formativas por si mismas, sino por el uso que se haga de ellas. Por todo ello, el condicionante principal del proceso de integración es el profesor, y principalmente el pensamiento que le guía, objeto de análisis que abordamos a continuación.

\section{ANÁlISIS DEL PENSAMIENTO DOCENTE: CREENCIAS, ACTITUDES Y EXPECTATIVAS}

Nos parece relevante señalar que una de las mejores formas de conocer sobre el pensamiento docente es a partir de las representaciones mentales. Éstas pueden ser definidas como "entidad de naturaleza cognitiva que refleja en el sistema mental de un individuo, una fracción del universo exterior a ese sistema" (Bloch \& Maturana, 1996:709). Se trata, por lo tanto, un modelo interiorizado de la persona surgido del ambiente que le permite adaptarse a él, propio de la cognición y experiencia del sujeto, así como de variables culturales y experiencias previas.

Las representaciones mentales se conforman por tres aspectos: En primer lugar, la información, es decir, la suma y organización de los conocimientos objetivos sobre el objeto de representación. En segundo lugar, la actitud o la orientación general, positiva o negativa frente al objeto de representación. Por último, el campo de representación o la idea de la organización del contenido en un todo unitario. Son difíciles de analizar debido a que sólo se pueden abordar mediante la puesta en juego de diversas inferencias que reflejen las intenciones de la persona, el mundo interno de la misma (Arranz, 2002a).

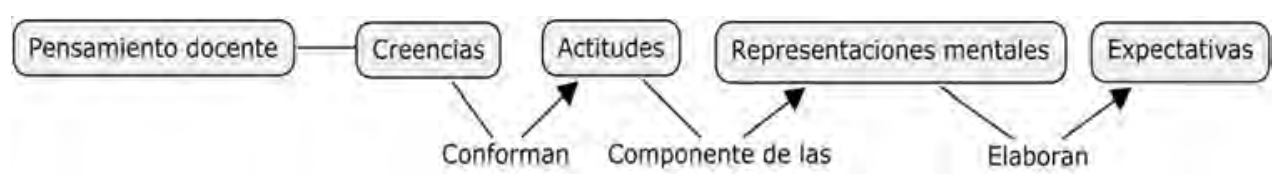

Figura 1. Factores del pensamiento docente relacionados con su actuación en el aula (Fuente: elaboración propia)

El análisis comienza por la base del pensamiento docente, es decir, las creencias. Éstas se pueden definir como aquellas proposiciones, premisas o procesos psicológicos que se valoran como fiables o ciertos (Richardson, 2003). Esto podría dar a entender que todo aquello que conocemos es lo que creemos, pero se debe indicar que "todo saber implica creencia, pero no toda creencia implica saber... Solemos pensar que sabemos cuando comprobamos que la creencia resulta verdadera, es decir, cuando la proposición en la que se expresa lo es" (Sola, 1999:663). Las creencias tienen una función muy marcada y necesaria, ya que sirven de guía o ayuda personal para definir y comprender, desde la propia persona, al mundo que la rodea.

En función de lo comentado, se podría inferir como "las creencias representan las concepciones educativas de los docentes en formación, las cuales van a organizar y estructurar su mundo profesional, influenciando al mismo tiempo sus acciones y decisiones" (Hervas \& Martín, 1996:2). Esto da muestra de cómo son la base fun- 
damental sobre la que se construye y se aplica toda innovación educativa, aunque sí que es cierto que éstas se relacionan con múltiples factores como el contexto o los conocimientos.

El concepto más relacionado con la idea de creencia es, sin duda, el otro aspecto destacado de las representaciones mentales, las actitudes, ya que surge de ambas. Se les denomina como "tendencia o disposición adquirida y relativamente duradera a evaluar de un modo determinado una persona, un objeto, un suceso o situación y a actuar en consecuencia con dicha evaluación" (Sarabia, 1992). La palabra clave es la adquisición, que connota una identidad no innata sino aprendido de la sociedad o entorno de la persona, indicando el camino a seguir en su trabajo y modificación ya que, como el contexto y la realidad, evolucionan.

Las actitudes se encuentran condicionadas por tres componentes valorativos: en primer lugar, el componente emocional o los sentimientos. En segundo, el componente conductual o cómo se actúa conforme a ello y, finalmente, el componente cognitivo o lo que se cree, influenciado éste por las creencias subjetivas y las objetivas que derivan del mencionado conjunto de información o conocimientos. Las tres se relacionan y son valoraciones de aquellas experiencias que se han presentado a lo largo de la vida y conforman una red experiencial determinada en función de las propias valoraciones y las de aquellas personas que sean similares a la red de actitudes (Arranz, 2002b).

Las experiencias permiten que las personas tengan una imagen e impresión acerca del resto de personas y cómo van a comportarse y actuar en función de ello (Vega \& Isidro, 1996). Las actitudes, por lo tanto, desempeñan diferentes funciones, como recogen Verdugo, Jenaro y Arias (1995):

- Función de entendimiento o satisfacción del conocimiento: Permiten la mejor comprensión e interpretación del entorno, unos automatismos que incluso desempeñan una función de supervivencia permitiendo reaccionar ante situaciones desconocidas mediante los estereotipos.

- Función egodefensiva: de la misma forma que ayudan en el entorno, nos protegen al permitir actuar defensivamente ante los ataques de este.

- Función utilitaria o de ajuste: permiten la adquisición de aquellos objetivos pretendidos al encaminar hacia los objetos con feedback positivo, por lo que nos ayudan a mejorar y progresar.

- Función facilitadora de la expresión de nuestros valores fundamentales: Aquellas funciones que no encaminan a un determinado fin actúan como medios de cara a la consecución del desarrollo personal mediante la adhesión a los valores buscados.

- Función de separación: actitudes que permiten mantener la distancia de aquellos elementos objeto de prejuicio, retroalimentándolas.

Como se ha visto hasta ahora, el pensamiento del docente se conforma de la creencia objetiva o conocimiento así como de la creencia subjetiva. Ambas se unifican en la formación del componente cognitivo que junto al componente emocional y el conductual dan lugar a las actitudes. Estas, junto con el conocimiento y el campo de 
representación, dan lugar a las representaciones mentales de la persona, en este caso docente, las cuales dan lugar a un nuevo aspecto a tener en cuenta: las expectativas.

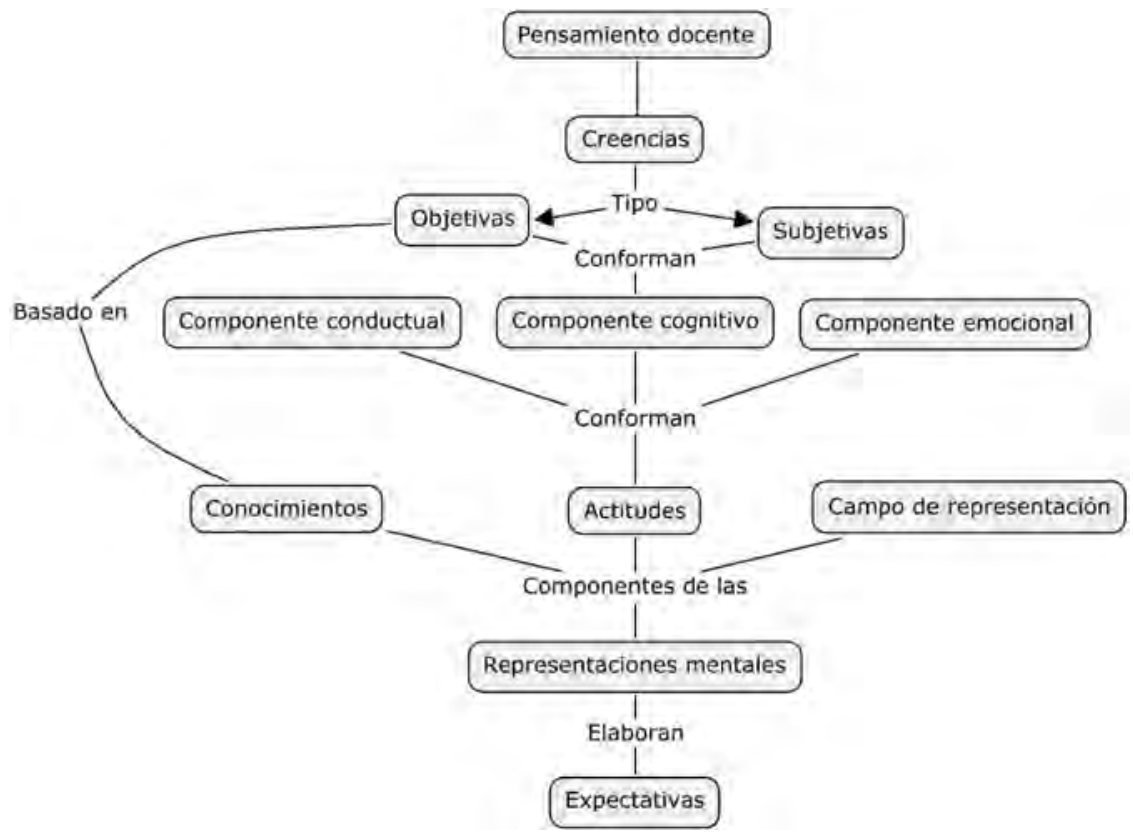

Figura 2. Componentes del pensamiento docente

(Fuente: adaptación propia basada en la de Arranz (2002b))

La perspectiva adquirida sobre el funcionamiento del pensamiento docente adolece, desde nuestro punto de vista, de un sesgo, ya que falta definir el funcionamiento del mismo. Las personas tienen la percepción orientada sólo hacia aquello que concuerda con sus preconcepciones, potenciando éstas y oponiéndose al cambio. Estamos en la línea de Vega e Isidro, (1996:2) al señalar que "las personas tienen tendencia a no cambiar sus expectativas a pesar de que reciban información incongruente con ellas y procesan mas la información positiva que la negativa".

En síntesis y valorando las aportaciones de Hervás \& Martín (1996), tres supuestos básicos son los que definen las actitudes y creencias del profesorado, las cuales son relevantes de cara a mejorar su propia formación:

- Las personas y los docentes, en especial los que se encuentran en formación, presentan concepciones sobre las que se fundamentan sus acciones mediante una interpretación de su significado.

- Los orígenes de las concepciones son diversos así como sus características, desde la idealización a las creencias.

- Lo que los docentes hacen se ve muy influenciado por aquello que piensan y cómo lo piensan, por lo que pocas acciones son espontáneas. (Yinger, 1986) 
Por todo ello, queda fuera de toda duda la importancia y funcionamiento que presenta el pensamiento, las percepciones, las actitudes, las creencias y las expectativas en la actuación del docente.

\section{El PENSAMIENTO DOCENTE Y SU INFLUENCIA EN LAS TICS: CLAVES PARA LA ACCIÓN}

Como se recoge en la literatura y ha quedado patente, el pensamiento docente expuesto, es también un factor determinante del uso de las TICs en el aula junto con la personalidad docente (Hoban, 2002), llegando a depender el uso y aprendizaje de las TIC de su adaptación o adecuación a las prácticas y creencias de los docentes sobre la enseñanza de su materia (Adell \& Gisbert, 1997). Esta importancia radica tanto en el aspecto positivo como negativo. En este sentido, un punto de vista positivo es expresado como una mejor integración de las TIC en el aula (Giamalas \& Nikolopoulou, 2010), mientras que una percepción o creencia de las TICs como pérdida de tiempo, o como un elemento estresante en el proceso educativo, conlleva una resistencia y valoración negativa ante el uso de las TICs.

Por su parte, esta valoración está muy relacionada con las creencias pedagógicas o perfil docente. Como indican Tondeur, Hermans, van Braak y Valcke, (2008), un perfil con altas creencias constructivistas utiliza frecuentemente el ordenador. En contraposición, aquel que presenta creencias tradicionales es menos dado al uso de las TIC en el aula, debido a que la acción principal del proceso es la interacción entre el estudiante y la persona que domina el contenido. Por último, los docentes con un pensamiento mixto presentan un mayor abanico de usos del ordenador que los otros por la mezcla de estilos educativos del docente.

Dentro del pensamiento docente, por otro lado, un aspecto fundamental son las creencias de autoeficacia. Estas se pueden definir como "las creencias en las propias capacidades para organizar y ejecutar los cursos de acción requeridos para producir determinados logros." (Bandura, 1997). Pese a que en la autoeficacia generalizada o "la confianza global en una habilidad de afrontamiento alrededor de un amplio rango de situaciones nuevas o demandas" (Schwarzer, 1999), podría quedar definido el uso de las TICs, existe un concepto propio: autoeficacia tecnológica. La consideración de las propias capacidades personales en el uso de las TICs fue definida como "una percepción de una de sus capacidades con respecto a habilidades y conocimientos específicos informáticos" (Murphy, Coover \& Owen, 1989).

La autoeficacia tecnológica presenta principalmente dos aspectos fundamentales. Por un lado, puede influir o afectar positivamente a una redefinición de los roles tanto del docente como del alumno, ya que las TICs pueden fomentar una transición del rol tradicional a uno en el que el docente desempeñe la función de facilitador del aprendizaje en el aula y, por ende, un papel más activo de los alumnos, en lugar de la tradicional consideración de receptores del aprendizaje, y por ello está relacionado con las creencias constructivistas mencionadas. Por otro, influye de forma determinante en el desarrollo de importantes herramientas educativas basadas en las TICs, aunque depende en cierto modo del resto de aspectos relacionados con el concepto como la autoimagen del docente, género, edad o experiencia previa. (Paraskeva, Bouta \& Papagianni, 2008) 
Este cambio de rol, depende mucho de que las valoraciones o creencias de los docentes, desemboquen en una serie de actitudes positivas o negativas ante las TIC o tecnoactitudes. Estas variarán gradualmente entre los dos grandes polos o extremos que existen: la tecnofobia o animadversión ante el uso de las TIC y la tecnofilia, plena afinidad o integración absoluta en el mundo tecnológico y el progreso que éstas representan. Las actitudes tan positivas hacia la TICs, son las que más peso tienen en el uso de las mismas en las aulas, por ello, algunos autores las señalan como la razón fundamental para el éxito de las inversiones en TIC. Esto es así debido a que el presentar unas actitudes tan predispuestas a su uso, desembocara en acciones o comportamientos reales en el aula en cuanto se le suministre al docente, además el docente estará motivado a la creación de herramientas y actividades para una efectiva aplicación en el curriculum que se aplique al aprendizaje del alumno (Teczi, 2009).

Queda por reflejar las causas que desembocan en unas actitudes negativas hacia el uso de las TICs en el aula, con el objetivo de evitarlas o desarrollar programas o acciones para trabajarlas. La principal proviene del mero hecho de modificar el rol tradicional del docente, que genera un desconcierto que implica una resistencia al cambio (Gisbert, 2000). Por otra parte, se recogen otras tres causas que tienen su presencia e importancia. La primera, expone el hecho de que no haya evidencias sobre la efectividad real del uso de las computadoras en el aprendizaje, como no hay una fundamentación basada en la experiencia de docentes que la hayan probado con éxito, le cuesta creer o adquirir creencias positivas de su uso y por lo tanto no genera una actitud abierta ante ellas sino tensión, indiferencia y/o rechazo. La segunda es la falta de conocimiento técnico del hardware y software, lo que conlleva a unas bajas creencias en las propias capacidades personales o una baja autoeficacia tecnológica que dificulta y entorpece el desarrollo de unas adecuadas actitudes, sin embargo fomenta la animadversión o miedo ante ellas. Por último, la falta de tiempo, medios y recursos percibida por el docente, le conduce al estrés y a la ansiedad al no poder atender a las demandas que las TICs le exigen, lo que obviamente le conduce al rechazo y evitación. (Tejedor, García-Valcárcel \& Prada, 2009).

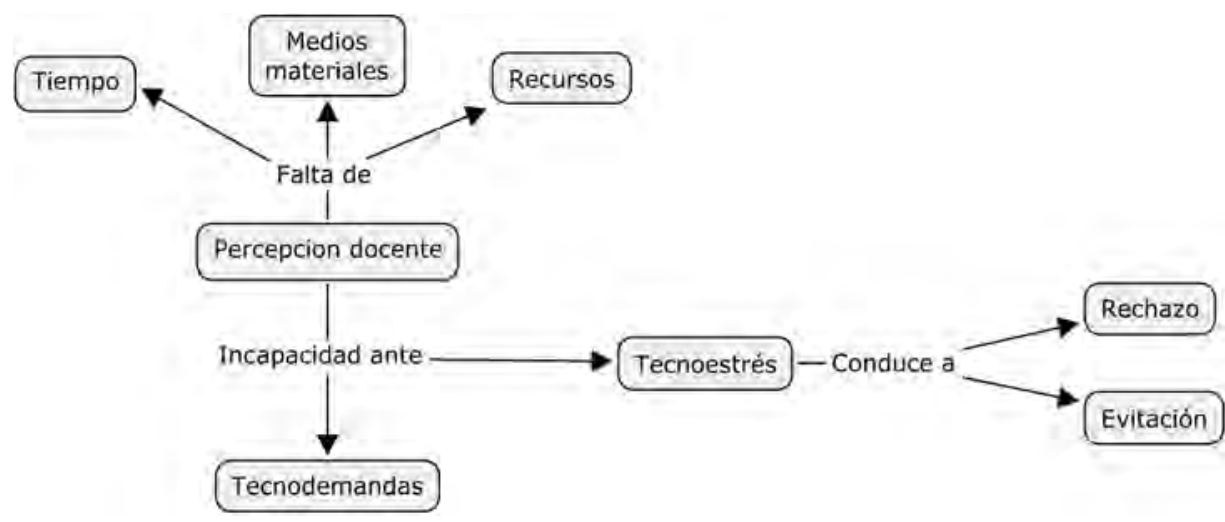

Figura 3. Factores que afectan a la integración de las TIC (Fuente: elaboración propia) 
Todas estas actitudes y/o creencias que mantiene el docente le conducen a presentar una serie de expectativas ante su uso. Si tiene una consideración positiva de las TICs, las expectativas que tenga de su utilización tendrán la misma orientación y estará mucho mas predispuesto a superar las dificultades existentes. Esto se debe a la motivación que le proporcionan las expectativas al docente de la mejora sustancial que va a representar para el alumno aquello que va a hacer. Por lo tanto, si un docente tiene unas altas expectativas ante el uso de las TIC en el aula, esta no sólo será posible, sino que será un éxito debido a que el docente realmente creerá que lo es. Del mismo modo, ocurre el efecto inverso, que como se ha tratado en el punto de partida, unas creencias y expectativas negativas dan lugar a unas tecnoactitudes negativas o lo que es lo mismo, la principal barrera para la implementación de las TICs en el aula.

El principal configurador de estas y otras creencias se encuentra en las enseñanzas recibidas, es por ello que aquello que los alumnos del grado de Magisterio vivencien en el aula, afectará a la configuración de su pensamiento docente y por lo tanto a su futura labor docente. De este modo y recogiendo tres puntos que Yang \& Huang (2008) recogen como necesarios para un pensamiento positivo, se pueden desarrollar dichos pasos o intervenciones que ayudarían al desarrollo de unas creencias positivas:

1. El primer paso consiste en facilitar unas explicaciones y demostraciones mas claras de los beneficios de las TIC para la enseñanza y el aprendizaje que redunden en unas experiencias poistivas que den lugar a unas creencias positivas de su aplicación debido a que se conocen buenos resultados y por lo tanto se configure una buena actitud y gama de expectativas.

2. El segundo paso se asienta en la dotación de incentivos a los docentes para integrar la tecnologia en el curriculum, motivarles o animarles. En determinados casos, sólo sería necesario tanto la dotación de material, que ya existe en determinadas etapas, como la dedicación de un tiempo específico al desarroIlo de la metodología con las TICs, de tal forma que fuera tiempo valorado y considerado.

3. Por último, se tendría que dotar de mucha más información relacionada con las TIC, no sólo en forma de cursillos o programas de formación permanente, sino también con una mayor presencia de las TICs en el curriculum educativo. Esta mayor competencia del docente radicaría en una positiva y sólida autoeficacia tecnológica que repercturía positivamente en el resto de componentes del pensamiento docente como se ha enunciado previamente.

\section{CONCLUSIONES}

Consideramos relevante señalar, en primer lugar, que el pensamiento centrado en las creencias, actitudes y expectativas es el principal motor del docente, la llave maestra que activa o no, en este caso, la implantación de las TICs en la escuela. Dicha implantación tiene sentido en la medida en que mejora la calidad del proceso de enseñanza-aprendizaje, superando las limitaciones de considerar una innovación educativa, simplemente, el hecho de presentar determinados contenidos con 
dichas tecnologías. Es fundamental, en este sentido, centrarse en una adecuada integración de las TICs, más allá de quedarse en seguir otorgando dotaciones a nivel de hardware a los centros.

Con una sólida integración positiva en sus creencias, facilitada por una alta autoeficacia percibida en su manejo y unas actitudes receptivas hacia la tecnología, se conseguirá cada vez con mayor calidad una escuela inclusiva, potenciadora de las competencias básicas, facilitando entornos de aprendizaje más motivadores y enriquecedores para los estudiantes. Resulta imprescindible revisar las políticas educativas y la inversión que se hace en esta materia, para dedicar los recursos humanos y económicos de la manera más eficiente posible, al tiempo que se dote al personal docente de las herramientas básicas, sobre todo, referidas a la formación, para evitar cierto riesgo de aumentar lo que hemos descrito como tecnoestrés, aspecto que puede abrir interesantes y necesarias líneas de investigación y actuación.

La aplicación de las TIC al proceso de enseñanza-aprendizaje brinda un reto a los formadores en la medida en que se plantea una educación íntegra del estudiante que le lleve hacia una completa emancipación para desempeñar un papel crítico y activo como ciudadano en la compleja sociedad actual. Es un proceso innovador y clave, en la medida que implica una transformacion sustancial de la concepción del aprendizaje, manteniendo los aspectos necesarios de los procesos de memorización, pero remarcando la comprensión a través de la interactividad, la simulación y la experimentación en una red globalizada de recursos, aspectos estos últimos que los puede proporcionar de una manera destacadas las TIC.

Hoy más que nunca, para conseguir estos cambios metodológicos y la mejora en la calidad del proceso de enseñanza-aprendizaje, es capital involucrar activamente a los docentes, y reivindicar su papel y participación en el debate por dicha calidad educativa. En este sentido, las implicaciones que presenta a las diferentes instituciones formativas de cara al profesorado son enormes. El Espacio Europeo de Educación Superior es una oportunidad para afianzar los aspectos positivos relativos a la formación de los maestros, tal y como se ha hecho históricamente, pero también para incorporar metodologías innovadoras, con las que se cuestione y mejore la calidad de los aprendizajes de los estudiantes, así como la necesaria interdisciplinariedad para que las TICs aporten todo el potencial que tienen. En este proceso formativo, resulta primordial fomentar unas creencias más constructivas y positivas que mejoren las actitudes del profesorado hacia las TICs.

Con este objetivo en perspectiva, es vital, por consiguiente, analizar el papel que representan las TICs en el curriculum de los actuales Grados de Magisterio y en la formación en el Master de Formación del Profesorado de Educación Secundaria, abriendo nuevas líneas de investigación para fundamentar y desarrollar programas para la mejora de las actitudes, expectativas y creencias de los docentes y alumnos del grado, en las TICs. Por último, otro camino abierto para seguir investigando es el de las actitudes, creencias y expectativas de los estudiantes, parte implicada en la innovación y centro del proceso de enseñanza-aprendizaje mediante la utilización de las TICs de forma crítica, selectiva y creativa.

Finalmente, la gran potencialidad de esta temática, ofrece posibilidades de desarrollar una futura línea de trabajo para complementar esta fundamentación teórica, 
basado en una investigación empírica que desarrolle un análisis de las diversas barreras institucionales desde diversos enfoques metodológicos. De esta forma, con la formación creciente del profesorado y jugando éste un papel capital en e proceso, permitirá salir a la escuela del retraso generacional que presenta: una institución del siglo XIX, con maestros formados, mayoritariamente, en el siglo XX y alumnos del siglo XXI.

\section{REFERENCIAS BIBLIOGRÁFICAS}

ABRAMI, P. C. (2001). Understanding and promoting complex learning using technology. Educational Research and Evaluation 7, 113-136.

AL-SENAIDI, S., LIN, L. y POIROT, J. (2009). Barriers to adopting technology for teaching and learning in Oman. Computers \& Education 53, 575-590.

ANGELI, C. y VALANIDES, N. (2009). Epistemological and methodological issues for the conceptualization, development, and assessment of ICT-TPCK: Advances in technological pedagogical content knowledge (TPCK). Computers \& Education 52, 154-168.

ARRANZ, P. (2002a). Representaciones mentales del profesorado respecto al síndrome de Down: Implicaciones para un cambio en los modelos de formación. En S. Molina, Psicopedagogía del niño con síndrome de Down (págs. 299-354). Maracena, Granada: Arial.

ARRANZ, P. (2002b): Las representaciones mentales de los maestros en ejercicio y en formación respecto al síndrome de Down. Tesis doctoral inédita. Universidad de Zaragoza.

BANDURA, A. (1987). Pensamiento y Acción. Barcelona: Martínez Roca.

BANDURA, A. (1997). Self-efficacy: The exercise of control. New York, NY: Freeman.

BAYLOR, A. y RITCHIE, D. (2002). What factors facilitate teacher skill, teacher morale, and perceived student learning in technology-using classrooms? Computers \& Education 39 (1), 395-414.

BLOCH, S. y MATURANA, H. (1996) "Biología del Emocionar y Alba Emoting, Bailando Juntos". Santiago de Chile: Dolmen.

CABERO, J. (2001). Utilización de recursos y medios en los procesos de enseñanzaaprendizaje. Actas de las IV Jornadas Nacionales de Desarrollo Curricular, Organizativo profesional. Jaén.

CABERO, J. (2008). TICs para la igualdad: la brecha digital en la discapacidad. Anales de la Universidad Metropolitana 8 (2), 15-43

CARACENA, J. M. (2007). La identidad virtual y el trabajo colaborativo en red como bases para el cambio de paradigma en la formación permanente del profesorado. Revista DIM: Didáctica, Innovación y Multimedia 10.

CHING, S. y HUANG, Y.-F. (2008). A study of high school English teacher's behavior, concerns and beliefs in integrating information technology into English instruction. Computers in human behavior 24 (3), 1085-1103.

DEVOS, G., BOUCKENOOGHE, D., ENGELS, N., HOTTON, G. y AELTERMAN, A. (2007). An assessment of well-being of principals in Flemish primary schools. Journal of Educational Administration 45, 33-61. 
DOOMEKAMP, B. (1994). A implementaçao de Computadores no Ensino Secundario: O exemplo da Holanda. Revista da Escola Superior de Educaçao de Portoalegre 16, 102-114.

ERTMER, P. (1999). Examining teachers' beliefs about the role of technology in the elementary classroom. Journal of Research on Computing in Education 32, 54-72.

ESTEBAN, M. (2005). La Educación Permanente y las Nuevas Tecnologías ante las necesidades educativas actuales. Quaderns digitals. Monográfico educación comparada 38, 1-9.

GIALAMAS, V. y NIKOLOPOULOU, K. (2010). In-service and pre-service early childhood teachers' views and intentions about ICT use in early childhood settings: A comparative study. Computers \& Education (Articulo en prensa).

GILSTER, P. (1997). Digital Literacy. New York: Wiley Computer Publishing.

GISBERT, M. (2000). El profesor del siglo XXI: De transmisor de contenidos a guía del ciberespacio. En Cabero y otros (Coords) (Ed.), Y continuamos avanzando. Las nuevas tecnologías para la mejora educativa (pp. 315-330). Sevilla: Kronos.

GÓMEZ, J., MUR, J. y SANZ, J. (2010). Redes Sociales en educación: El camino hacia la escuela 2.0. Actas del II Congreso Galego de Investigación en Ciencias da Educación. Santiago de Compostela.

GONZÁLEZ, A. P. (1998). Másallá del curriculum: La educación ante el reto de las nuevas tecnologías de la información y la comunicación. Universitas Tarraconensis: Revista De Ciències De l'Educació 22, 151-162.

HAWKRIDGE, D. (1990). Who needs computers in school, and why? Computers and Education 15, 1-6.

HERVÁS, C. y MARTÍN, J. (1997). Evaluación de necesidades formativas, actitudes y creencias del profesorado de Educación Secundaria. Revista Electrónica Interuniversitaria de Formación del Profesorado 1 (0).

HOBAN, G. (2002). Teacher learning for educational change. Buckingham: Open University Press.

HUGHES, M. y ZACHARIAH, S. (2001). An investigation into the relationship between effective administrative leadership styles and the use of technology. International Electronic Journal for Leadership in Learning 5, 1-10.

HULPIA, H. y VALCKE, M. (2004). The use of performance indicators in a school improvement policy: The theoretical and empirical context. Evaluation \& Research in Education 18, 102-120.

IGLESIAS, F., VEIGA, E. y GONZÁLEZ, M. (2000). Actitudes del profesorado de primaria hacia la informática: un estudio exploratorio. $V$ Congreso Galego-Portugués de Psicopedagoxía Actas (Comunicacións e Posters) 6 (4), 258-265.

LAJOIE, S. P. (2003). Individual differences in spatial ability: developing technologies to increase strategy awareness and skills. Educational Psychologist 38, 115-125.

LI-PING, T. y AUSTIN, M. J. (2009). Students' perceptions of teaching technologies, application of technologies, and academic performance. Computers \& Education 53, 1241-1255.

MAC BEATH, J. (1999). Schools Must Speak for Themselves: the Case for School Selfevaluation. London: Routledge. 
MARQUÉS, P. (2008). El impacto de las TIC en educación: funciones y limitaciones. Recuperado el 30 de marzo de 2010, de: http://peremarques.pangea.org/ siyedu.htm.

MUELLER, J., WOOD, E., WILLOUGHBY, T., ROSS, C. y SPECHT, J. (2008). Identifying discriminating variables between teachers who fully integrate computers and teachers with limited integration. Computers \& Education 51, 1523-1537.

MURPHY, C. A., COOVER, D. y OWEN, S. V. (1989). Development and validation of the computer self-efficacy scale. Educational and Pyschological Measurement 49, 893-899.

OTTO, T. L. y ALBION, P. R. (2002). Understanding the role of school leaders in realizing the potential of ICTs in education. International conference of the association for the advancement of computing in education. Nashville.

PARASKEVA, F., BOUTA, H. y PAPAGIANI, A. (2008). Individual characteristics and computer self-efficacy in secondary education teachers to integrate technology in educational practice. Computers \& Education 50 (3), 1084-1091.

PERRENOUD, P. (2002), Construir competencias desde la escuela, Dolmen ediciones, Santiago de Chile, Chile, p. 23.

PRENSKY, M. (2001). Digital Natives, Digital Immigrants. On the Horizon 9 (5), 1-6. RICHARDSON, V. (2003). Constructivist pedagogy. Teachers College Record 105, 1623-1640.

ROSEN, L. y WEIL, M. (1995). Computer availability, computer experience and technophobia among public school teachers. Computers in Human Behavior 11, 9-31.

SEFTON-GREEN, J. (2006). Youth, technology, and media cultures. Review of Research in Education 30, 279-306.

SNOEYINK, R. y ERTMER, P. (2001). Thrust into technology: How veteran teachers respond. Journal of Educational Technology Systems 30, 85-111.

SUTHERLAND, R., ARMSTRONG, V., BARNES, S., BRAWN, R., BREEZE, N. y GALL, M. (2004). Transforming teaching and learning: Embedding ICT into everyday classroom practices. Journal of Computer Assisted Learning 20, 413-425.

TANG, P. S. y ANG, P. H. (2002). The diffusion of information technology in Singapore schools: A process framework. New Media \& Society 4, 457-478.

TEJEDOR, F. J., GARCÍA-VALCÁRCEL, A. y PRADA, S. (2009). Medida de actitudes del profesoradouniversitariohacia la integración de las TIC. Comunicar 17 (33), 115-124.

TEZCI, E. (2009). Teachers' effect on ict use in education: the Turkey sample. Procedia Social and Behavioral Sciences 1, 1285-1294.

TONDEUR, J., VAN KEER, H., VAN BRAAK, J. y VALCKE, M. (2008). ICT integration in the classroom: Challenging the potential of a school policy. Computers \& Education 51, 212-223.

TRIGGS, P. y JOHN, P. (2004). From transaction to transformation: Information and communication technology, professional development and the formation of communities of practice. Journal of Computer Assisted Learning 20, 416-439. 
VEEN, W. (1993). The role of beliefs in the use of information technology: Implications for teacher education, or teaching the right thing at the right time. Journal of Information Technology for Teacher Education 2, 139-153.

VEGA, M. T. y ISIDRO, A. I. (1997). Las creencias académico sociales del profesor y sus efectos. Revista Electrónica Interuniversitaria de Formación del Profesorado, $1(0)$.

VERDUGO, M. A., JENARO, C. y ARIAS, B. (1995). "Actitudes sociales y profesionales hacia las personas con discapacidad: estrategias de evaluación e intervención", en M. A. VERDUGO y A. AGUADO: Personas con discapacidad: perspectivas psicopedagógicas y rehabilitadoras. México, Siglo XXI, pp. 79-143.

YANG, S. C. y HUANG, Y. (2008). A study of high school english teachers' behavior, concerns and beliefs in integrating information technology into english instruction. Computers in Human Behavior, 24 (3), 1085-1103. 